is generated in the nodules or in the adjacent neocortex. The heterotopia are part of an abnormal circuitry involving surrounding cortex.

EEG-fMRI studies of grey matter heterotopia at the Montreal Neurological Institute showed metabolic responses in the heterotopia while spikes were generated in the neocortex (Kobayashi E et al. Brain 2006;129:366-374). Activation responses reflected excitation involving the heterotopia and surrounding cortex, and deactivation also reflected a distant, extra-lesional inhibition. EEG-fMRI is a non-invasive procedure that may explain the epileptogenicity of neuronal migration disorders and the involvement of areas of the brain distant from the heterotopia.

\title{
ANGELMAN SYNDROME AND EPILEPSY
}

Twenty-six patients with Angelman syndrome (AS), of which 19 had 15q11-13 maternal deletion, were studied and followed at the University of San Paulo, Brazil, with particular reference to the prevalence and type of epilepsy and its response to antiepileptic drugs. Epilepsy occurred in 22 patients (85\%), 19 with deletion and 3 without positive genetic confirmation. All 19 patients with deletion had generalized seizures, and 10 (53\%) had partial seizures. Video-EEG uncovered atypical absence and subtle myoclonic seizures, often missed by parents; it also recorded nonconvulsive status that is sometimes prolonged and associated with cognitive decline in 7 . Mean age at onset of seizures was 1 year 1 month, and in 18 patients, epilepsy preceded the clinical diagnosis of AS. Five (26\%) had their first seizure with fever, and $10(53 \%)$ had epilepsy aggravated by fever. Sixteen $(84 \%)$ had status epilepticus, associated with hyperthermia in 7. Valproic acid, alone or in combination with phenobarbital or clonazepam, improved seizure control while carbamazepine, oxcarbazepine, and vigabatrin caused aggravation of seizures. Refractory seizures occurred in $16(84 \%)$ during infancy and early childhood, but seizure frequency decreased at a mean age of 5.3 years. Improvement in seizure frequency and severity continued through late childhood and puberty. (Valente KD, Koiffmann CP, Fridman C, et al. Epilepsy in patients with Angelman syndrome caused by deletion of the chromosome 15q11-13. Arch Neurol January 2006;63:122-128). (Respond: Kette D Valente MD PhD, Department of Psychiatry, University of Sao Paulo, R Jesuino Arruda, 901/51, 04532-082 Sao Paulo, SP Brazil).

COMMENT. Angelman syndrome is characterized by developmental delay, severe mental retardation, paroxysmal laughter, ataxia associated with hand flapping, seizures, and stereotyped jerky movements, regarded as cortical myoclonus (Guerrini R et al. Ann Neurol 1996;40:39-48). Physical signs include microcephaly, prominent jaw, wide mouth, pointed chin, and hypopigmentation. Seizures are mainly generalized, frequently partial, atypical absence, myoclonic, sometimes included as a cause of West, or Lennox-Gastaut syndromes, and, as shown in the above study, often febrile in type.

The typical interictal EEG abnormality shows rhythmic slow waves at $4-6 \mathrm{~Hz}$ and runs of $2-3 \mathrm{~Hz}$ slow spike-wave complexes anteriorly. Eye closure is accompanied by spikes and 2-4 Hz slow waves posteriorly (Robb SA et al. Arch Dis Child 1989;64:83-86; Sugimoto T et al. Epilepsia 1992;33:1078-1082). Status epilepticus is a frequent complication, sometimes precipitated by hyperthermia, and often recurrent. Age-related improvement in seizure frequency and severity is an additional characteristic. 
The diagnosis of AS should be considered in infants with developmental delay and generalized cryptogenic seizures resistant to medication. Diagnosis is confirmed in $85 \%$ by genetic testing and deletion of chromosome 15q11-13. In rare cases, other genetic mechanisms have been uncovered, and in $15 \%$ of cases, diagnosis is made on clinical and EEG findings alone. Where possible, the genetic cause should be determined as a prelude to family counseling.

\section{RISK OF EMERGENCY INTRACRANIAL PATHOLOGY AFTER FIRST COMPLEX FEBRILE SEIZURE}

The outcome of 71 neurologically normal children after a first complex febrile seizure (CFS) was determined by review of neuroimaging findings, telephone interviews, and medical records, in a prospective study at Columbia University College of Physicians and Surgeons, New York. During the study period March 1999-July 2002, 293 children presented to the ED with a first febrile seizure and of these, $79(27 \%)$ were complex in type. Complex characteristics (focal, multiple episodes in a 24 hour period, and prolonged duration $>15 \mathrm{~min}$ ) were single in $51(72 \%)$, of which 20 were focal, 22 multiple episodes, and 9 prolonged CFS. Twenty had seizures with multiple complex features. Lumbar puncture was performed in $10(14 \%)$ in the ED, and none had meningitis. Emergency cranial CT scans were obtained in the ED for 10 patients (14\%), and $13(18 \%)$ patients were admitted. A total of $46(65 \%)$ patients had emergency CT scans in the ED and/or MRI scans within 1 week after the ED visit and were followed prospectively. Outcome was determined by telephone interview within the subsequent 4 to 44 months (median 22.4 months) in 12 (17\%) patients and by medical record review in $13(18 \%)$. Of the 71 patients studied, none had intracranial pathology requiring emergency medical or neurosurgical intervention $(95 \%$ confidence interval: $4 \%$ ). It is concluded that the risk of emergency intracranial pathology following a CFS is low, and routine emergency neuroimaging in children with first complex febrile seizures is probably unnecessary. (Teng D, Dayan P, Tyler S, et al. Risk of intracranial pathologic conditions requiring emergency intervention after a first complex febrile seizure episode among children. Pediatrics February 2006;117:304-308). (Respond: Dale C Hesdorffer PhD, Gertrude H Sergievsky Center, Columbia University, $630 \mathrm{~W} 168^{\text {th }}$ St, P\&S Unit 16, New York, NY 10032).

COMMENT. In children presenting with a complex febrile seizure (CFS), each patient must be evaluated clinically to exclude possible meningitis, encephalitis or abscess, and routine neuroimaging in the ED is considered unnecessary. CT scan is not without risk, both from radiation and the need for sedation. Risk factors for subsequent epilepsy in children with FS include the CFS, a family history of epilepsy, and neurodevelopmental abnormality (in Commentary by DiMario FJ Jr. Pediatrics 2006;117:528-530); adapted from Shinnar S et al. J Child Neurol 2002;17(suppl 1):S44-S52). Earlier studies found that the duration of the FS was the most important determinant of the later development of epilepsy and an abnormal EEG (Millichap JG et al. Neurology 1960;10:643-653; Maher J et al. Brain 1995;118:1521-1528). Neuroimaging should be considered in the individual patient with a CFS that is very prolonged or is followed by a persistent epileptiform abnormality in the EEG. 